\title{
Suppression of cell proliferation and collagen production in cultured human hypertrophic scar fibroblasts by Sp1 decoy oligodeoxynucleotide
}

\author{
CHENLIANG DENG, JIANGHONG ZHENG, WEIDONG WAN, SHIXIN ZHANG, \\ ZHI DING, GUANGYU MAO and SONGLIN YANG
}

Department of Plastic Surgery, Shanghai 6th People's Hospital, Shanghai Jiaotong University, Shanghai 200233, P.R. China

Received September 19, 2012; Accepted January 4, 2013

DOI: $10.3892 / \mathrm{mmr} .2013 .1278$

\begin{abstract}
Hypertrophic scars are characterized by the abnormal proliferation of fibroblasts and an overproduction of collagen. The Sp1 transcription factor is involved in the stimulation of collagen synthesis. A decoy oligonucleotide (ODN) targeting Sp1 was designed and transfected into hypertrophic scar fibroblasts (HSFs) cells using cationic liposomes. The transfection efficiency was determined by flow cytometry and was observed to be $85 \pm 7 \%(n=5)$. Specific binding of the Sp1 decoy ODN was monitored with an electrophoretic mobility shift assay (EMSA). Following transfection with the decoy ODN to Sp1, cell viability and cell proliferation, which were examined by the cell counting kit WST-8, were decreased by $80 \%$ compared with untreated cells. Transforming growth factor- $\beta$ (TGF- $\beta$ ) mRNA and collagen mRNA expression were also reduced by $48 \%$ in the transfection decoy ODN group. The cell viability of HSFs after 48 h of transfection with 25,50, 100 and $150 \mathrm{nM} \mathrm{Sp1} \mathrm{decoy}$ ODN was $0.9331 \pm 0.0203,0.7479 \pm 0.0868,0.577 \pm 0.0347$ and $0.4703 \pm 0.0147$, respectively. The $100 \mathrm{nM}$ dose of the $\mathrm{Sp} 1$ decoy ODN inhibited the expression of types I and III collagen by 32 and $28 \%$, respectively (both $\mathrm{P}<0.01$ ). TGF- $\beta$ mRNA expression was also effectively suppressed by the $100 \mathrm{nM}$ Sp1 decoy ODN $(\mathrm{P}<0.01)$. The Sp1 decoy ODN inhibited cell proliferation and the expression of types I and III collagen. Therefore, Sp1 decoy ODNs may be a promising tool for developing and testing novel therapeutic applications for treating hypertrophic scars.
\end{abstract}

\section{Introduction}

Hypertrophic scars are a malformation frequently ecountered by plastic surgeons. Characteristic morphological and ultrastructural changes in hypertrophic scars include the abnormal

Correspondence to: Professor Songlin Yang, Department of Plastic Surgery, Shanghai 6th People's Hospital, Shanghai Jiaotong University, Yi Shan Road 600, Shanghai 200233, P.R. China

E-mail: alcee@126.com; yangsonglin5803@163.com

Key words: decoy oligodeoxynucleotide, transcription factor, cell proliferation, collagen, fibroblast and excessive deposition of extracellular matrix (ECM). Excessive scar fibrosis results from the increased expression of several ECM proteins, including collagen. The collagen genes pro-COL1A1 and pro-COL1A2 are responsible for the synthesis of pro1 and pro2 polypeptides of type I collagen and they are synthesized at a ratio of $2: 1$ to form a stable triple helical type I collagen molecule in the extracellular space (1). Fibrosis, the excessive accumulation of collagen, is induced by persistent pro-fibrotic growth factor signaling, including fibroblast growth factor (FGF), platelet-derived growth factor (PDGF) and, most predominantly, transforming growth factor- $\beta 1$ (TGF- $\beta 1)$. Sp1 is a transcription factor that regulates the expression of a variety of ECM genes and is involved in numerous growth factor-related signal transduction pathways, including TGF- $\beta 1$, FGF and PDGF signaling (2).

$\mathrm{Sp} 1$ belongs to a family of ubiquitous transcription factors associated with GC-rich promoters that are inducible and involved in inflammation (3). Sp1 exists in a variety of isoforms which bind with varying affinities to consensus sequences that form a transcriptional network to fine-tune gene expression. Sp1-dependent transcription is altered during cell growth through increased gene expression.

Treating fibroblasts with TGF- $\beta 1$ promotes the synthesis of type I collagen (4). Chronic exposure to TGF- $\beta 1$ in lungs increases the deposition of type I collagen, causing excessive fibrosis, which may increase morbidity and mortality. Direct silencing of the COL1A1 gene is one method to reduce collagen type I synthesis. A novel double-stranded (ds) DNA decoy with phosphorothioate (PT) linkages containing the TGF- $\beta$ cis-element has been observed in the distal promoter region of the COL1A1 gene (5). This decoy blocks collagen type I synthesis in human hypertrophic scar fibroblasts (HSFs) stimulated by TGF- $\beta 1$ (6). These findings support the concept that a decoy ODN may inhibit collagen type I synthesis at the transcriptional and translational levels. Therefore, we performed this ex vivo study to determine whether a Sp1 decoy ODN could inhibit HSF proliferation, TGF- $\beta$ signaling and collagen production.

\section{Materials and methods}

Cell culture. Control human fibroblasts were isolated from normal human skin (the individual biopsies ranged in size 
from 80 to $150 \mathrm{~mm}^{3} ; \mathrm{n}=5$ ). The size of hypertrophic scars used for culture ranged from 500 to $1,300 \mathrm{~mm}^{3}(\mathrm{n}=5)$. The samples were collected at the Shanghai 6th People's Hospital (Shanghai, China) after approval by the ethics committee for human studies. The patients provided informed consent and the patient clinical characteristics are listed in Table I. None of the patients had a systemic disease and none had been previously treated for the scars. Primary human fibroblasts were isolated from each hypertrophic scar sample before scar tissue was fixed in formalin for routine histological examination. The tissue sections were cut into 1-3 $\mathrm{mm}$ cubes and incubated with $200 \mathrm{U} / \mathrm{ml}$ type I collagenase for $4 \mathrm{~h}$ at $37^{\circ} \mathrm{C}$. The cell cultures were maintained in Dulbecco's modified Eagle's medium (DMEM) supplemented with $10 \%$ fetal bovine serum (FBS), $2 \mathrm{mM}$ glutamine, $100 \mathrm{U} / \mathrm{ml}$ penicillin and $100 \mathrm{mg} / \mathrm{ml}$ streptomycin at $37^{\circ} \mathrm{C}$ in a humidified incubator with $5 \% \mathrm{CO}_{2}$. HSF cultures were analyzed at passages 3-8.

Synthesis and modification of the Sp1 decoy ODNs. The sense ODN containing the binding sequence for Sp1 was synthesized and PT-modified at the first and last three bases. The antisense ODN was 5-carboxyfluorescein (5'-FAM) and 3'-biotin-labeled (Bio Basic Inc., Shanghai, China). The ds PT decoy ODNs were prepared by annealing the sense and antisense strands in $200 \mathrm{mM} \mathrm{NaCl}$ at $95^{\circ} \mathrm{C}$ for $7 \mathrm{~min}$ and slowly cooling to $4^{\circ} \mathrm{C}$. These were designated Sp1 decoy ODNs.

Mutated Sp1 decoy ODNs (designated mut-Sp1 decoy ODNs) were mutated at two positions (shown as lowercase letters) in the Sp1 binding sequence of the Sp1 decoy ODNs. Inspection of the resulting nucleotide sequences showed no sequence homology to other known transcription factors by searching databases on transcriptional regulation for $\mathrm{Sp} 1$ decoy ODNs (5'-gccccgatcttttgatcggggeggggcgagcttttg ctcgeccc-3') and scrambled-Sp1 decoy ODNs (5'-ctgactgacttttta gtcagtcagtcagtcagtcttttcagtcagtca-3').

Spl decoy ODN distribution test. Control fibroblasts and HSFs were plated on glass coverslips and the coverslips were put into 35-mm dishes in DMEM containing 10\% FBS. When the cells reached 60-70\% confluence, the cells were serum-starved for $24 \mathrm{~h}$ in DMEM containing 0.5\% FBS and transfected with FAM-labeled-Sp1 decoy ODNs. Briefly, $1 \mu \mathrm{g}$ of the Sp1 decoy ODNs were mixed with 3-6 $\mu$ l of lipofectamine plus reagent (Invitrogen, Carlsbad, CA, USA) and transfected with modified supplier instructions. The Sp1 decoy ODNs and liposome mixtures were added into each well and incubated at $37^{\circ} \mathrm{C}$ for $3,6,12,24$ and $48 \mathrm{~h}$. The HSFs were stained with 4',6-diamidino-2-phenylindole (DAPI) and observed by laser scanning confocal fluorescence microscopy.

ODN transfection. We transfected Sp1 decoy ODNs into HSFs using the cationic liposome method. Briefly, HSFs were seeded on a 6-well plate and cultured in DMEM containing 10\% FBS with antibiotics. When the cells reached $80-90 \%$ confluence, the cells were transfected with $10 \mathrm{nM} \mathrm{Sp1}$ and the scrambled Sp1 decoy-ODNs in Lipofectamine Plus (0.7 $\mu$ g DNA: $2 \mu$ 1 lipid; Invitrogen). For the decoy ODN, lipid mixture was added to the cells according to the manufacturer's instructions. Following incubation at $37^{\circ} \mathrm{C}$ for $4 \mathrm{~h}$, the cells were cultured in fresh medium with $10 \%$ serum and maintained until use.
Table I. Patient characteristics.

\begin{tabular}{lllc}
\hline Age (years) & Gender & Biopsy site & Duration (years) \\
\hline 32 & Male & Chest & 3 \\
32 & Male & Shoulder & 4 \\
18 & Female & Earlobe & 1 \\
21 & Female & Earlobe & 2 \\
28 & Female & Chest & 3 \\
\hline
\end{tabular}

Cytotoxicity assay of HSFs. HSFs were seeded in a 96-well plate $\left(5 \times 10^{3}\right.$ cells/well) and cultured in DMEM containing $10 \%$ FBS. After $24 \mathrm{~h}$, the cells were transfected with Sp1 or scrambled Sp1 decoy ODNs. Briefly, 10, 50, 100 or $200 \mathrm{nM} \mathrm{Sp1}$ or scrambled Spl ODNs was mixed with Lipofectamine Plus reagent (Invitrogen) and transfected with modified supplier instructions. After $48 \mathrm{~h}$, a WST-8 cell viability assay was performed with the Cell Counting kit-8 (Dojindo, Kumamoto, Japan) according to the manufacturer's instructions. HSFs that were not transfected with cationic liposomes were used as controls. The experiments were replicated in triplicate.

Cell proliferation analysis. Cultured HSF proliferation was measured using the Cell Counting kit-8 (Dojindo). HSFs were rendered quiescent by incubation for $24 \mathrm{~h}$ in serum-free media. To evaluate the effect of Sp1 decoy ODNs on HSF proliferation, Lipofectamine Plus and Sp1 decoy ODNs (100 nM) were added to the wells and the cells were incubated at $37^{\circ} \mathrm{C}$ for an additional $4 \mathrm{~h}$. The cell proliferation index was determined after $48 \mathrm{~h}$. Cells without transfection of scrambled Sp1 decoy ODNs were used as controls. Each experiment was performed in triplicate. Control fibroblast proliferation was measured with the Cell Counting kit-8.

Electrophoretic mobility shift assays (EMSAs). EMSAs were performed on nuclear extracts prepared from the control and HSFs using the ds PT decoy ODNs containing the Sp1 binding site. The LightShift Chemiluminescent EMSA kit (Pierce, Rockford, IL, USA) was used. In brief, 20 fmol decoy ODN probes, with 3'-end labeled with biotin, were incubated with $1 \mu \mathrm{g}$ nuclear extract, $1 \mu \mathrm{g} / \mu \mathrm{l}$ poly(dI:dC), $50 \mathrm{mM} \mathrm{NaCl}$, $200 \mathrm{mM}$ EDTA, $1 \mathrm{M} \mathrm{KCl}, 100 \mathrm{mM} \mathrm{MgCl}_{2}, 1 \% \mathrm{NP}-40$ and $50 \%$ glycerol. After incubation, the samples were separated on $6 \%$ native polyacrylamide gels with a $1 \mathrm{X}$ Tris-borate-EDTA (TBE) running buffer $(450 \mathrm{mM}$ Tris, $450 \mathrm{mM}$ boric acid, $10 \mathrm{mM}$ EDTA, pH 8.3) and biotin-labeled DNA was detected by chemiluminescence. For competition experiments, 100 -fold molar excess of the unlabeled probe was added to the reaction mixture before the addition of the labeled probe.

RNA isolation and reverse transcription-polymerase chain reaction ( $R T-P C R)$. Total RNA was isolated from confluent fibroblasts using TRIzol reagent (Invitrogen) and the integrity of the RNA was determined by agarose gel electrophoresis. For the RT-PCR, $2 \mu \mathrm{g}$ total RNA was reverse transcribed at $37^{\circ} \mathrm{C}$ for $1 \mathrm{~h}$ in a $25-\mu 1$ reaction volume containing $250 \mathrm{mM}$ Tris-HCl, $375 \mathrm{mM} \mathrm{KCl}, 15 \mathrm{mM} \mathrm{MgCl}_{2}, 50 \mathrm{mM}$ dithiothreitol, $10 \mathrm{mM}$ dNTPs, $0.5 \mu \mathrm{g}$ oligo (DT) 20 primer, 100 units reverse 
Table II. Primer design and product lengths for RT-PCR products.

\begin{tabular}{llr}
\hline Gene & \multicolumn{1}{c}{ Primer } & Length (bp) \\
\hline COL1A1 & 5-AAAGACGGGAGGGCGAGTG-3 & 242 \\
COL1A2 & 5-GCCATAGGACATCTGGGAAGCAA-3 & 288 \\
COL3A1 & 5-CTCCAAGGAAATGGCAACTCA-3 & 58 \\
FN & 5-AGGAACGGCAGGCGAGAT-3 & 241 \\
& 5-CCCACAGCCTTCTTCTACACCT-3 & 249 \\
GAPDH & 5-ACCCATTCCTCCCACTCC-3 & \\
& 5-GCCGAATGTAGATGAGGAG-3 & 280 \\
\hline
\end{tabular}

RT-PCR, reverse transcription-polymerase chain reaction; bp, base pair; COL1A1, collagen I $\alpha 1$; COL1A2, collagen I $\alpha 2$; COL3A1, collagen III $\alpha 1$; FN, fibronectin; GAPDH, glyceraldehyde-3-phosphate dehydrogenase.

A

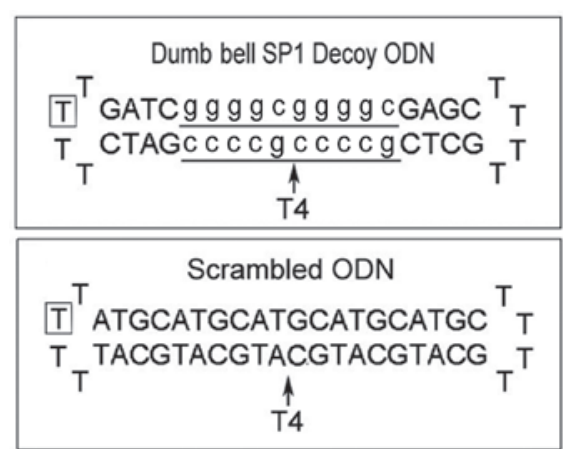

Figure 1. (A) Dumbbell Sp1 decoy oligonucleotide (ODN) and (B) scrambled ODN. The transcription factor Sp1 binding site is underlined. The arrow indicates the gap connected by T4 DNA ligase after annealing and the box indicates the site labeled by carboxyfluorescein (FAM).

transcriptase Moloney Murine Leukemia Virus (reverse transcriptase M-MLV) and 25 units ribonuclease inhibitor (Takara Bio, Inc., Shiga, Japan) and subjected to PCR amplification with the primers listed in Table II. Glyceraldehyde-3-phosphate dehydrogenase (GAPDH) was amplified as the internal control. RT products $(0.5-1 \mu \mathrm{g})$ were amplified with 1 unit Taq DNA polymerase (Takara Bio, Inc.) and $1 \mathrm{mM}$ of each primer in $50 \mu \mathrm{l}$ of reaction mix containing $50 \mathrm{mM} \mathrm{KCl}, 10 \mathrm{mM}$ Tris- $\mathrm{HCl}$, $1.5 \mathrm{mM} \mathrm{MgCl}_{2}$ and $0.02 \mathrm{mM}$ each of dNTPs as follows: initial denaturation for $3 \mathrm{~min}$ at $94^{\circ} \mathrm{C}, 30$ cycles of amplification, 1 min of denaturation at $94^{\circ} \mathrm{C}$, different annealing temperatures for each pair of primers, 1 min of extension at $72^{\circ} \mathrm{C}$ and a final elongation for $5 \mathrm{~min}$ at $72^{\circ} \mathrm{C}$. Parallel RT-PCR assays without reverse transcriptase were performed for each sample to confirm that the PCR products resulted from cDNA rather than from genomic DNA. The PCR products $(10 \mu \mathrm{l})$ were analyzed by $2 \%$ agarose gel electrophoresis. Relative mRNA abundance was calculated by densitometric analysis using computer software (Kodak Digital Science 1D Image Analysis software; Eastman Kodak Co., Rochester, NY, USA).

Statistical analysis. Statistical Package for Social Sciences (SPSS, Inc., Chicago, IL, USA), version 13.0, was used for

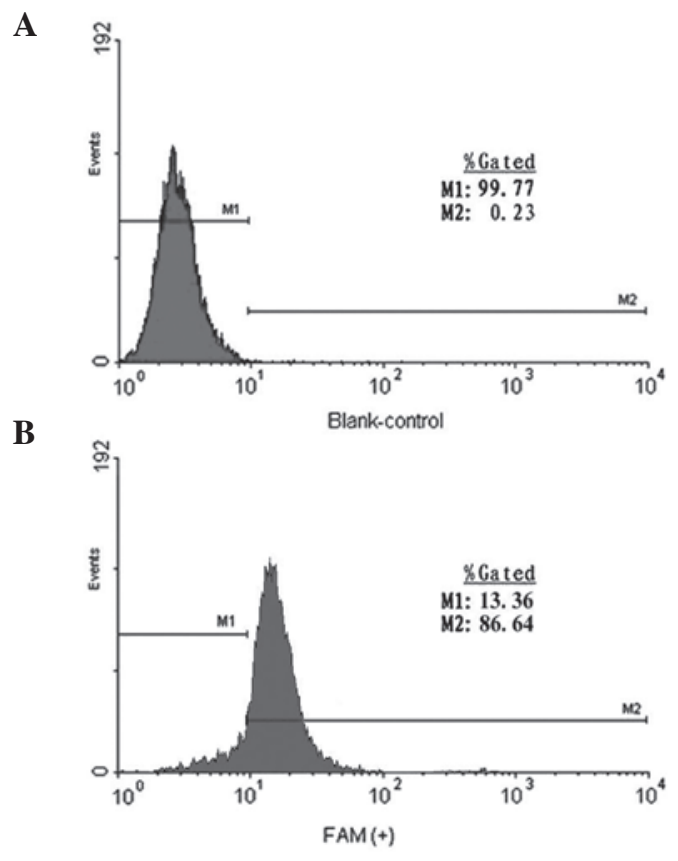

Figure 2. Transfection efficiency of the cells transfected with $100 \mathrm{nM}$ carboxyfluorescein (FAM)-labeled oligonucleotides (ODNs). (A) Blank control. (B) FAM-labeled ODN in HSFs. The transfection efficiency was $>99 \%$ at $4 \mathrm{~h}$ after ODN transfection. HSFs, hypertrophic scar fibroblasts.

statistical analyses and the Wilcoxon signed rank test was used to determine if there were differences between the groups. $\mathrm{P}<0.05$ was considered to indicate a statistically significant result.

\section{Results}

Design and identification of Spl decoy ODNs. Dumbbell Sp1 decoy ODNs and scrambled Sp1 ODNs were designed and the structure and sequence of the ODNs are shown in Fig. 1. Cells transfected with $100 \mathrm{nM}$ FAM-labeled ODNs showed positive results by flow cytometry, with the positive efficiency being $>99 \%$. No fluorescence was detected in cells transfected with the blank control (Fig. 2). 


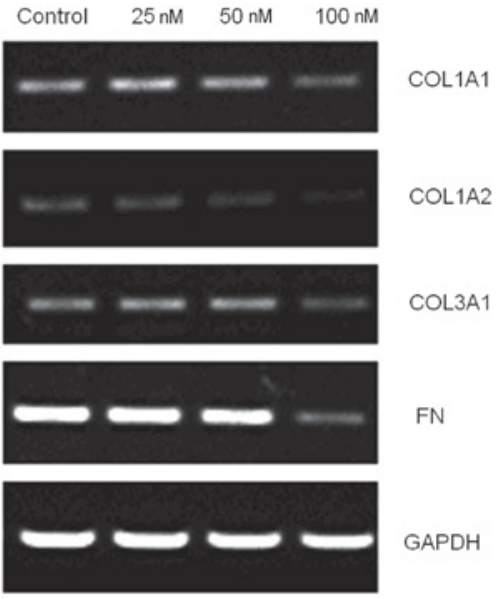

Sp1 Decoy ODN

Figure 3. Extracelluar matrix mRNA expression was inhibited by the $\mathrm{Sp} 1$ decoy oligonucleotide (ODN). COL1A1, collagen I $\alpha 1$; COL1A2, collagen I $\alpha 2$; COL3A1, collagen III $\alpha 1$; FN, fibronectin; GAPDH, glyceraldehyde3-phosphate dehydrogenase.
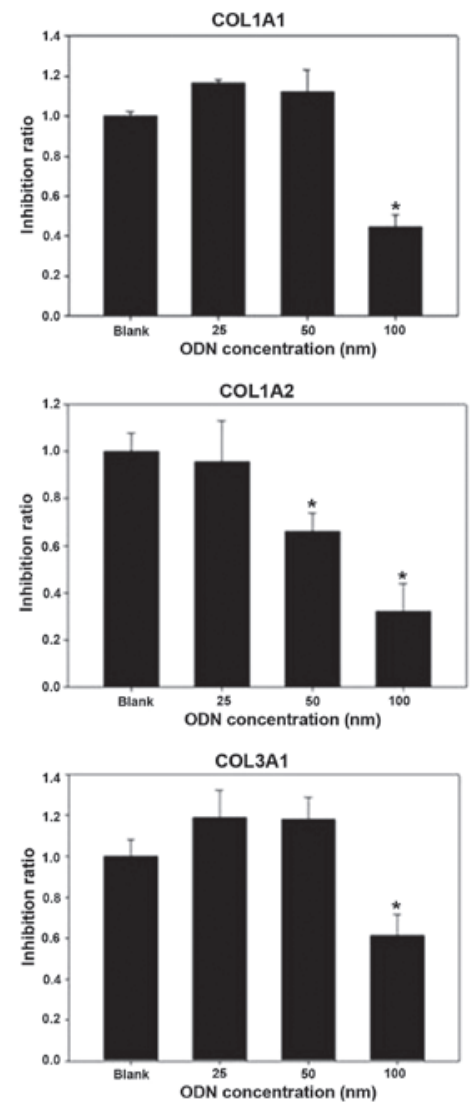

FN

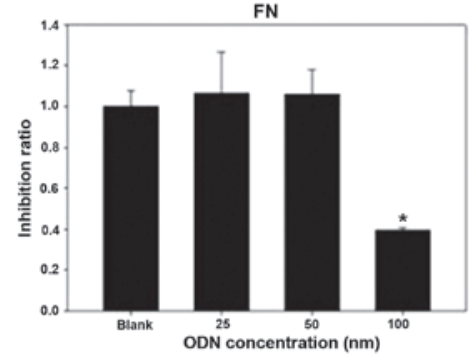

Figure 4. Effects of 25, 50 and $100 \mathrm{nM} \mathrm{Sp1} \mathrm{decoy} \mathrm{oligonucleotides} \mathrm{(ODNs)} \mathrm{on}$ COL1A1, COL1A2, COL3A1 and fibronectin (FN) expression. The sample size was $n=3$ per group for each concentration; ${ }^{*} \mathrm{P}<0.05$ vs. the blank control.

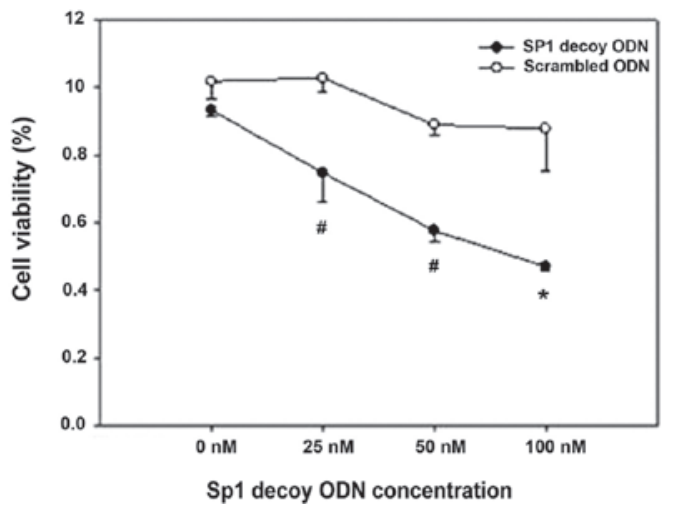

Figure 5. Cell viability was reduced in hypertrophic scar fibroblasts (HSFs) exposed to increasing concentrations of the Sp1 decoy oligonucleotide (ODN). There were no significant differences with the scrambled ODN control. The sample size was $\mathrm{n}=5$ per group for each concentration. ${ }^{*} \mathrm{P}<0.05$ vs. $0 \mathrm{nM}$ and ${ }^{\text {"}} \mathrm{P}<0.05$ vs. the respective scambled ODN.

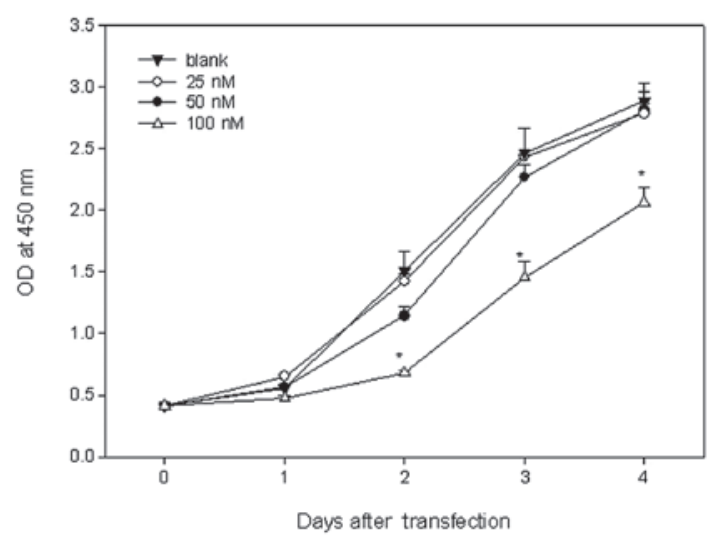

Figure 6. Cell proliferation was reduced in hypertrophic scar fibroblasts (HSFs) transfected with $100 \mathrm{nM}$ of the Sp1 decoy oligonucleotide (ODN). The sample size was $n=8$ per group for each concentration. " $\mathrm{P}<0.05$ compared with the blank control.

Inhibition of collagen type I and III expression by SpI decoy ODNs. At $48 \mathrm{~h}$ after transfection with Sp1 decoy ODNs, COL1A1 and COL3A1 mRNA levels were significantly reduced (both $\mathrm{P}<0.01$; Fig. 3). However, the control ODNs had no inhibitory effect on the expression of collagen type I or III (Fig. 4; P>0.05).

Suppression of cell proliferation by Spl decoy ODNs. Cultured HSFs were transfected with FAM-labeled ODNs targeting Sp1 transcriptional factor, with the negative control containing a random sequence. Transfection with $100 \mathrm{nM} \mathrm{Sp1} \mathrm{decoy}$ ODN resulted in minimal cell viability (Fig. 5). There were significant differences between cells transfected with the Sp1 decoy ODN and scrambled ODN at the 25, 50 and $100 \mathrm{nM}$ doses. No significant difference was observed between cells transfected with the Sp1 decoy ODN and scrambled Sp1 ODN effect at $0 \mathrm{nM}(\mathrm{P}=0.21)$. HSFs treated with $100 \mathrm{nM} \mathrm{Sp1} \mathrm{ODN}$ had minimal growth potential. There were significant differences between cells treated with $100 \mathrm{nM} \mathrm{Sp1} \mathrm{ODN} \mathrm{and} \mathrm{the}$ other concentrations at 2 days after transfection. There were no significant differences between the cells treated with the 25 and $50 \mathrm{nM}$ dose ( $\mathrm{P}=0.23$; Fig. 6). 


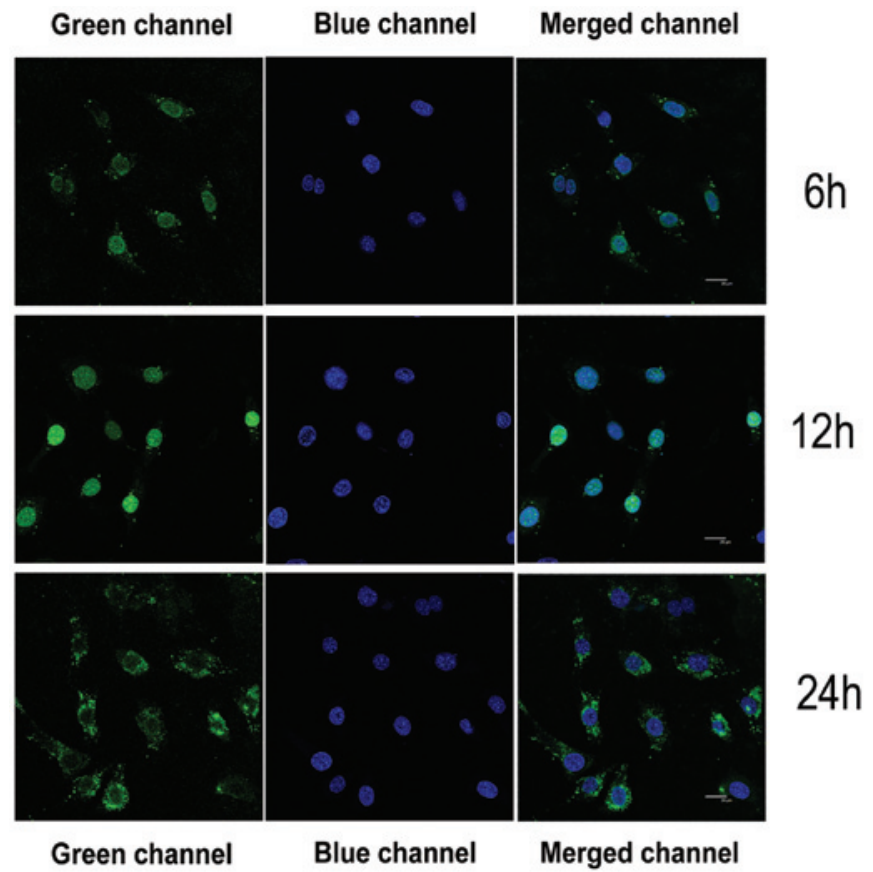

Figure 7. Hypertrophic scar fibroblasts (HSFs) were transfected with the $100 \mathrm{nM} \mathrm{Sp1}$ decoy oligonucleotide (ODN) and imaged at 6, 12 and $24 \mathrm{~h}$ after transfection. At $12 \mathrm{~h}$ post-transfection, the yellow green fluorescence appeared in the cell nuclei of merged channel (yellow green fluorescence is a result of green and blue screen overlay), while at $24 \mathrm{~h}$ post-transfection the green fluorescence was observed in the cytoplasm of the infected cells.

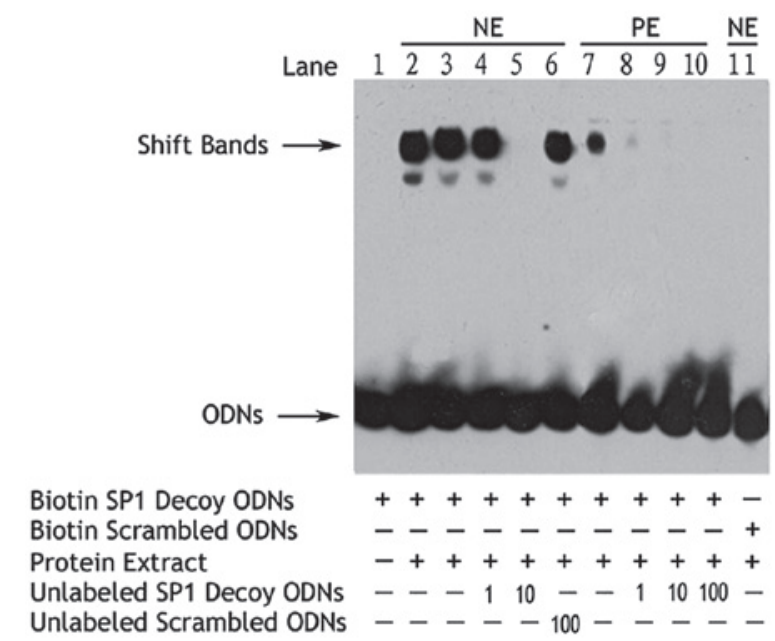

Figure 8. In vitro, nuclear extracts (NE; lanes 2-6 and 11) and plasma extracts (PE; lanes 7-10) were added to the biotin-labeled Sp1 decoy oligonucleotide (ODN) probe and co-cultured at room temperature before electrophoresis Bands that were shifted are shown in lanes 2 and 3. A 10 -fold increase in unlabeled Sp1 decoy ODN completely inhibited the binding of nuclear proteins and biotin-labeled Sp1 decoy ODN (lane 5). A 100-fold increase in unlabeled scrambled ODN showed no inhibition effect (lane 6). Biotinlabeled scrambled ODN could not bind with the nuclear protein extracts (lane 11).

Location of Spl decoy ODNs in cells after transfection. Yellow-green fluorescence appeared in the cell nucleus after HSFs were transfected with the $100 \mathrm{nM}$ Sp1 decoy ODN for 6-12 h. By $24 \mathrm{~h}$ post-transfection, the yellow-green fluorescence was observed in the cytoplasm of the infected cells (Fig. 7).

The EMSA assay revealed that a 10 -fold increase in the unlabeled Spl decoy ODN inhibited binding of the nuclear protein and biotin-labeled Sp1 decoy ODN (Fig. 8). A 100-fold increase in the unlabeled scrambled Sp1 ODN showed no inhibitory effect, while the biotin-labeled scrambled Sp1 ODN was unable to bind nuclear protein.

\section{Discussion}

Enhanced HSF proliferation and increased collagen production are principal contributors to hypertrophic scar formation (7). This process is governed by signal transduction cascades that are finely regulated by growth factors. The transcription factor Sp1 is ubiquitously expressed and plays a significant role in signal transduction (8). The most significant findings of the current study were that the Sp1 decoy ODN inhibited cell proliferation and collagen expression. These results demonstrate that Sp1 decoy ODNs may provide a promising therapeutic option for the treatment of hypertrophic scars.

Complementary approaches have also demonstrated that Sp1 targeting may be a potentially powerful therapeutic approach for reducing ECM accumulation in hypertrophic scars (9). Recently, a number of new technologies have been developed to inhibit target gene expression in a sequence-specific manner and these technologies have been investigated as treatment modalities for a variety of diseases $(10,11)$. While there are numerous studies on strategies targeting mRNA via antisense ODNs, ribozymes and RNA interference, targeting proteins that regulate expression of a specific gene is a relatively novel approach (12). Decoy technology has recently been developed in an attempt to reduce the activity of a specific transcription factor through the use of cis-element ds ODNs containing the consensus binding sequence of the transcription factor (13).

The greatest restriction to using ODNs is that they are easily degraded by nucleases or by readily nonspecific reac- 
tions with the control strand. To circumvent these problems, various modified DNA analogs, particularly ODNs with PT-modified ODNs, are often employed. These modified DNA analogs replace the oxygen atoms in the phosphodiester bond of the terminal nucleotides with sulfur atoms. This structural modification increases resistance to nuclease attack (14). In this study, we used dumbbell Sp1 decoy ODNs to enhance the stability of the decoy. Using this method, Sp1 regulated the expression of collagen. Similar approaches have been used to deliver gene-regulating molecules, such as synthetic ds ODNs that mimic cis-acting promoter elements $(15,16)$.

In the present study, Sp1 decoy ODNs inhibited HSF proliferation and downregulated collagen synthesis. Sp1 decoy ODNs at concentrations above $10 \mathrm{nM}$ significantly inhibited HSF proliferation. In addition, the Sp1 decoy ODN suppressed TGF- $\beta 1$ and fibronectin mRNA levels, as well as cell proliferation induced by PDGF (12). EMSA evaluation showed that the DNA-protein complex induced by PDGF induction was reduced significantly in HSFs transfected with Sp1 decoy ODNs, whereas the scramble decoy ODNs had no effect on DNA binding activity. Using reporter plasmids containing the Sp1 binding motif in the TGF- $\beta 1$ and fibronectin promoters, the enhancement in Sp1-dependent transcription activity in response to serum stimulation was reduced significantly by the Sp1 decoy ODN (17).

We also demonstrated that the in vitro addition of the Sp1 decoy ODN effectively attenuated ECM mRNA induction and the resulting ECM deposition (18). Therefore, these results suggest that the Sp1 decoy ODN represents a potentially effective gene therapy strategy to ameliorate dermal ECM accumulation during hypertrophic scar formation (19). In addition, Sp1 decoy ODNs markedly suppressed collagen IV mRNA levels and fibronectin mRNA and protein in the hypertrophic scar (20).

One limitation of this study is that $\mathrm{Sp1}$ regulates numerous genes, many of which are not involved in the etiology of hypertrophic scars. The present study did not analyze any of these genes. While this limitation precludes the decoy ODN approach from being useful as a systemic therapeutic strategy, this approach could be viable as a topical ointment applied directly to the skin. Whether decoy ODNs would be useful for other diseases that involve Sp1 upregulation remains to be investigated.

In conclusion, our data demonstrate that $\mathrm{Sp} 1$ is a key transcription factor mediating HSF proliferation and ECM gene expression that may prevent the pathogenesis of the hypertrophic scar. Although additional in vitro and in vivo studies need to be conducted to fully understand the potential of decoy ODNs in preventing excessive collagen contraction in patients with hypertrophic scars, this study provides a platform for these experiments.

\section{Acknowledgements}

This study was supported by the National Scientific Fund (30571929, 81000837), through the Experimental Study Center, for the 6th People's Hospital at Shanghai Jiaotong University (Shanghai, China). We thank Medjaden Bioscience Limited for assisting in the preparation of this manuscript.

\section{References}

1. Wang R, Ghahary A, Shen Q, Scott PG, Roy K and Tredget EE: Hypertrophic scar tissues and fibroblasts produce more transforming growth factor-beta1 mRNA and protein than normal skin and cells. Wound Repair Regen 8: 128-137, 2000.

2. Jagadeesan $J$ and Bayat A: Transforming growth factor beta (TGFbeta) and keloid disease. Int J Surg 5: 278-285, 2007.

3. Chin GS, Liu W, Peled Z, et al: Differential expression of transforming growth factor-beta receptors I and II and activation of Smad 3 in keloid fibroblasts. Plast Reconstr Surg 108: 423-429, 2001.

4. Chen SJ, Artlett CM, Jimenez SA and Varga J: Modulation of human alpha1(I) procollagen gene activity by interaction with $\mathrm{Sp} 1$ and Sp3 transcription factors in vitro. Gene 215: 101-110, 1998.

5. Boros DL, Singh KP, Gerard HC, Hudson AP, White SL and Cutroneo KR: A novel nonsteroidal antifibrotic oligo decoy containing the TGF-beta element found in the COL1A1 gene which regulates murine schistosomiasis liver fibrosis. J Cell Physiol 204: 370-374, 2005.

6. Chen SJ, Yuan W, Lo S, Trojanowska M and Varga J: Interaction of smad3 with a proximal smad-binding element of the human alpha2(I) procollagen gene promoter required for transcriptional activation by TGF-beta. J Cell Physiol 183: 381-392, 2000.

7. Naim R, Naumann A, Barnes J, et al: Transforming growth factor-beta1-antisense modulates the expression of hepatocyte growth factor/scatter factor in keloid fibroblast cell culture. Aesthetic Plast Surg 32: 346-352, 2008.

8. Meisler NT, Chiu JF and Cutroneo KR: Promoter competitors as novel antifibrotics that inhibit transforming growth factor-beta induction of collagen and noncollagen protein synthesis in fibroblasts. J Cell Biochem 75: 196-205, 1999.

9. Bowley E, O'Gorman DB and Gan BS: Beta-catenin signaling in fibroproliferative disease. J Surg Res 138: 141-150, 2007.

10. Smith RA, Miller TM, Yamanaka K, et al: Antisense oligonucleotide therapy for neurodegenerative disease. J Clin Invest 116: 2290-2296, 2006.

11. Lindow M and Kauppinen S: Discovering the first microRNAtargeted drug. J Cell Biol 199: 407-412, 2012.

12. Sato M: Upregulation of the Wnt/beta-catenin pathway induced by transforming growth factor-beta in hypertrophic scars and keloids. Acta Derm Venereol 86: 300-307, 2006.

13. Takamizawa J, Konishi H, Yanagisawa K, et al: Reduced expression of the let-7 microRNAs in human lung cancers in association with shortened postoperative survival. Cancer Res 64: 3753-3756, 2004.

14. Calin GA, Dumitru CD, Shimizu M, et al: Frequent deletions and down-regulation of micro- RNA genes miR15 and miR16 at $13 q 14$ in chronic lymphocytic leukemia. Proc Natl Acad Sci USA 99: 15524-15529, 2002.

15. Ahn JD, Kim CH, Magae J, et al: E2F decoy oligodeoxynucleotides effectively inhibit growth of human tumor cells. Biochem Biophys Res Commun 310: 1048-1053, 2003.

16. Ahn JD, Morishita R, Kaneda Y, et al: Inhibitory effects of novel AP-1 decoy oligodeoxynucleotides on vascular smooth muscle cell proliferation in vitro and neointimal formation in vivo. Circ Res 90: 1325-1332, 2002.

17. Nusse R: Wnt signaling in disease and in development. Cell Res 15: 28-32, 2005 .

18. Kato M, Zhang J, Wang M, et al: MicroRNA-192 in diabetic kidney glomeruli and its function in TGF-beta-induced collagen expression via inhibition of E-box repressors. Proc Natl Acad Sci USA 104: 3432-3437, 2007.

19. Cheon SS, Wei Q, Gurung A, et al: Beta-catenin regulates wound size and mediates the effect of TGF-beta in cutaneous healing. FASEB J 20: 692-701, 2006.

20. Berezikov E, Guryev V, van de Belt J, Wienholds E, Plasterk RH and Cuppen E: Phylogenetic shadowing and computational identification of human microRNA genes. Cell 120: 21-24, 2005. 